Tropical Journal of Pharmaceutical Research February 2016; 15 (2): 355-361

ISSN: $1596-5996$ (print); 1596-9827 (electronic)

(C) Pharmacotherapy Group, Faculty of Pharmacy, University of Benin, Benin City, 300001 Nigeria.

All rights reserved.

Available online at http://www.tjpr.org

Original Research Article

http://dx.doi.org/10.4314/tjpr.v15i2.19

\title{
Effects of Kangshen Oral Liquid on Gentamicin-induced Acute Kidney Injury in Rats
}

\author{
Jing-Sheng Zhao ${ }^{1}$, Yuan-Liang Wei $^{2}$, Xin-Wei Lin ${ }^{1}$, Hong Liu ${ }^{3}$, Xue-Qing Xiao ${ }^{4}$, \\ Jun Yang ${ }^{1}$, Wei-Guo $\mathrm{Yi}^{5 *}$, Xiang-Nan $\mathrm{Li}^{6 *}$ and Dan $\mathrm{Hao}^{7}$ \\ ${ }^{1}$ Department of Heart-Kidney Internal Medicine, ${ }^{2}$ Department of Vasculocardiology, ${ }^{3}$ Department of Drug and Equipment \\ Section, ${ }^{4}$ Department of Nursing, ${ }^{5}$ Department of Medicine, The 152nd Central Hospital of PLA, Pingdingshan Henan 467000 , \\ ${ }^{6}$ Graduate School, Dalian Medical University, Dalian Liaoning 116044, ${ }^{7}$ Department of Oncology and Hematology, The 152nd \\ Central Hospital of PLA, Pingdingshan Henan 467000, PR China
}

*For correspondence: Email: weiguoyipla@sina.com; xiangnanlidlmu@sina.com

\begin{abstract}
Purpose: To investigate the effects of Kangshen oral liquid (KSOL) on gentamicin sulfate (GS)-induced acute kidney injury (AKI) in rats.

Methods: The rats were randomly divided into seven groups and treated with normal saline (NS), GS, furosemide, uremic clearance granule, or one of three different doses of KSOL. Several AKI-related indices were monitored, including renal index (RI), urine protein (UP) level, B-N-acetyl-glucosaminidase (NAG) activity in the urine, serum creatinine (SCr) level, blood urea nitrogen (BUN) level in the serum, malondialdehyde (MDA) level, and superoxide dismutase (SOD) activity in the kidney tissue. Histopathological changes in the kidney tissue of each group were monitored using light microscopy.

Results: After treatment with $K S O L$ (20, 10, or $5 \mathrm{~mL} / \mathrm{kg}$ ), the Rl, activity of $N A G$ and levels of UP, SCr, BUN, and MDA were significantly decreased by $6.66-41.47 \%$, and the activity of SOD was significantly increased by $15.85-20.27 \%$, compared with the GS group $(p<0.05$ or 0.01$)$. The histopathological studies showed that KSOL notably improved GS-induced atrophy of the glomeruli, lymphocytic infiltration in the interstitium of the cortex, protein deposits in the collecting tubules, and hyperemia of the renal interstitium.

Conclusion: KSOL improves GS-induced AKI at biochemical and histopathological levels, and thus has a potential to be developed into a therapeutic drug for AKI.
\end{abstract}

Keywords: Kangshen oral liquid, Gentamicin, Acute kidney injury, Furosemide, Uremic clearance granule, Biochemical parameters

Tropical Journal of Pharmaceutical Research is indexed by Science Citation Index (SciSearch), Scopus, International Pharmaceutical Abstract, Chemical Abstracts, Embase, Index Copernicus, EBSCO, African Index Medicus, JournalSeek, Journal Citation Reports/Science Edition, Directory of Open Access Journals (DOAJ), African Journal Online, Bioline International, Open-J-Gate and Pharmacy Abstracts

\section{INTRODUCTION}

Acute kidney injury (AKI) is a common and important diagnostic and therapeutic challenge for clinicians [1]. It is characterized by a rapid decrease of renal excretory function and the accumulation of products of nitrogen metabolism such as creatinine, urea, and other waste products. Because the AKI definition emphasizes the filtration function of the kidney, it can be easily and routinely diagnosed by analyzing the serum creatinine ( $\mathrm{SCr}$ ) and urea concentrations $[2,3]$.

The use of vasopressors and conditions such as diabetes mellitus, chronic renal disease, and sepsis are some triggers for AKI, while hypovolemia, creatinine on admission, and high 
severity scores are clinical indicators of the condition [4-6]. The mortality of AKI patients is 60 - $70 \%[7,8]$. About $20-40 \%$ of AKI patients die in the hospital, and the mortality rates are even higher in patients who suffer from severe AKI $[9,10]$. Currently, specific therapies have not been developed to prevent or cure AKI. Patients who survive AKI often suffer from chronic kidney disease or end-stage renal disease [11,12]. Therefore, it is critical to develop new, safe and effective drugs to prevent or treat AKI.

Based on the knowledge of the compatibility and characteristics of traditional Chinese medicines (TCMs), Kangshen oral liquid (KSOL), which consists of: Ginseng radix et rhizoma (150 g), Astragali radix $(200 \mathrm{~g}), \quad$ Atractylodis Macrocephalae rhizoma (150 g), Poria (200 g), Epimedii folium (150 g), Dioscoreae rhizoma $(150 \mathrm{~g})$, Pheretima (100 g), Carthami flos (200 g), Rosae Laevigatae fructus (200 g), Euryales semen $(100 \mathrm{~g})$, and Glycyrrhizae radix et rhizoma (40 g), was developed by Wei-Guo Yi, Xiang-Nan $\mathrm{Li}$ and their team to help patients with AKI. The aim of this study was to investigate the effects of KSOL on gentamicin sulfate (GS)treated $\mathrm{AKI}$ in rats by observing the histopathological changes of kidney tissue and determining AKI-related indices, such as the renal index (RI), the urine protein (UP) level, the $\mathrm{B}-\mathrm{N}$-acetyl-glucosaminidase (NAG) activity in the urine, the serum creatinine ( $\mathrm{SCr}$ ) and blood urea nitrogen (BUN) levels in the serum, as well as the malondialdehyde (MDA) level and superoxide dismutase (SOD) activity in the kidney tissue.

\section{EXPERIMENTAL}

\section{Plant materials}

Ginseng radix et rhizoma, Astragali radix, Atractylodis Macrocephalae rhizoma, Poria, Epimedii folium, Dioscoreae rhizoma, Pheretima, Carthami flos, Rosae Laevigatae fructus, Euryales semen, and Glycyrrhizae radix et rhizoma were purchased from Beijing TongRenTang Co., Ltd and identified by WeiGuo Yi, who work in Department of Medicine, the 152nd Central Hospital of PLA (Pingdingshan, China). Eleven voucher specimens for each crude drug (voucher nos. 152CHPLA2009010 152CHPLA2009020) were stored in Dalian Medical University (Liaoning, China) for future reference.

\section{Chemicals and reagents}

Uremic clearance granule (UCG) was purchased from Inner Mongolia Shankangchen
Pharmaceutical Industry (batch no. 20110114). Furosemide was obtained from Tianjin Lisheng Pharmaceutical Co., Ltd (batch no. 1101008). Normal saline (NS) was provided by Henan Tailing Pharmaceutical Co., Ltd., (batch no. 11082465). GS was manufactured by Henan Furen Huaiqingtang Pharmaceutical Co., Ltd., (batch no. 1108301). Formalin was purchased from Yantai Shuangshuang Chemical Co., Ltd., (batch no. 20110310). Acetic acid was obtained from Tianjin Hengxing Chemical Reagent Co., Ltd., (batch no. 20110114). The SCr assay kit (batch no. 20111223), urea assay kit (batch no. 20111223), UP test kit (batch no. 20120109), NAG assay kit (batch no. 20111201B), SOD assay kit (batch no. 20120105), and MDA assay kit (batch no. 20111227) were purchased from Nanjing Jiancheng Bioengineering Institute, China.

\section{Animals}

Adult Wistar rats $(200 \pm 20 \mathrm{~g})$ were obtained from Hebei Experimental Animal Center and housed in metabolic cages $\left(25^{\circ} \mathrm{C}, 12 \mathrm{~h}\right.$ light/dark cycle). All animals received water and food ad libitum. Animal treatments were conducted in strict accordance with the ethical guidelines of the National Institutes of Health Guide for the Care and Use of Laboratory Animals [13]. Experiments were carried out with the approval of the ethics committee of the 152nd Central Hospital of PLA (protocol no. 2009092632).

\section{Preparation of KSOL}

KSOL was provided by the 152nd Central Hospital of PLA's manufacturing laboratory (batch no. 20110425). KSOL was prepared as follows. According to the ratio described above, 11 kinds of TCMs were finely ground and boiled thrice with water for $40 \mathrm{~min}$. The combined decoction filtrate was concentrated under reduced pressure by rotary evaporators to reach a relative density of $1.15-1.30$ at $80{ }^{\circ} \mathrm{C}$, and then the concentrate was diluted with ethanol to obtain a $60 \%$ ethanol solution. The resulting solution was incubated standing at room temperature for $24 \mathrm{~h}$. After filtration, the ethanol solution was condensed under reduced pressure by rotary evaporators to remove the alcohol, and simple syrup was added into the solution. The above solution was diluted to $1000 \mathrm{~mL}(\mathrm{pH}=5.0$ - 7.0) with water, and then the diluted solution was filtrated and sterilized to produce KSOL.

\section{Grouping, modeling, and treating}

Eighty-four Wistar rats were randomly divided into seven groups $(n=12)$ to receive one of 
seven treatments: NS, GS, furosemide, UCG, or one of the three different doses of KSOL. Rats treated with GS, furosemide, UCG, or KSOL were first injected intraperitoneally with $140 \mathrm{mg}$ $\mathrm{GS} / \mathrm{kg}$ at 9:00 a.m. once a day for 7 days to establish the AKI model. Meanwhile at 6 p.m. each day, rats in the GS, furosemide, or UCG groups were separately administered orally with $\mathrm{NS}, 6.6 \mathrm{mg}$ furosemide $/ \mathrm{kg}$, or $4.2 \mathrm{~g} \mathrm{UCG} / \mathrm{kg}$, and rats in the KSOL groups were administered orally with high $(20 \mathrm{~mL} / \mathrm{kg})$, middle $(10 \mathrm{~mL} / \mathrm{kg})$, or low $(5 \mathrm{~mL} / \mathrm{kg}$ ) doses of $\mathrm{KSOL}$, respectively. Rats in the NS group were injected intraperitoneally with NS at 9:00 a.m. and administered orally with NS at 6 p.m. once a day for 7 days. All drugs were dissolved in NS or diluted with NS to obtain different concentrations such that the rats received an intragastric volume of $20 \mathrm{~mL} / \mathrm{kg}$.

After $1 \mathrm{~h}$ of drug treatment on 7th day, rats were transferred to metabolic cages, and the urine excreted by the rats was collected over $24 \mathrm{~h}$ and used to determine the NAG activity and UP level. Orbital blood was gathered on the 8th day and centrifuged at $3000 \mathrm{r} / \mathrm{min}$ for $10 \mathrm{~min}$ at $4{ }^{\circ} \mathrm{C}$ to obtain serum, which was used to determine the $\mathrm{SCr}$ and BUN levels. Subsequently, the rats were sacrificed by decapitation, and their kidneys were removed and washed with NS, blotted with a piece of filter paper and weighed. The right kidney was homogenized and centrifuged at $3000 \mathrm{r} / \mathrm{min}$ for $10 \mathrm{~min}$ at $4{ }^{\circ} \mathrm{C}$ to obtain the supernatant, which was used to analyze the SOD activity and MDA level. The left kidneys were isolated for histopathological investigation. RI was calculated as in Eq 1.

$\mathrm{RI}(\%)=(\mathrm{A} / \mathrm{B}) 100$

where $A$ and $B$ stand for the weight of the renal parenchyma and the body weight, respectively.

\section{Biochemical determinations}

The levels of UP, SCr, BUN, MDA, and the activities of NAG and SOD were determined using the corresponding kits according to the manufacturer's instructions for each [14-16]. After reactions were completed, absorbance was determined for all samples using a UV-visible spectrophotometer (Unico Shanghai Instrument Co. Ltd., China) or a 680Microplate Reader (BioRad, USA).

The detection wavelengths for UP, SCr, BUN, MDA, NAG, and SOD were set at 595, 510, 640, 640,450 , and $560 \mathrm{~nm}$, respectively. The absorbance for these indices was used to calculate the level or activity according to the corresponding standard curves.

\section{Histopathological studies}

Tissue fragments were taken from the left kidney tissue from representative rats in each group and fixed in $10 \%$ formalin. The fixed kidney tissue was washed six times with PBS, dehydrated in ethanol, and embedded in paraffin. The embedded tissue fragment was sliced at $3 \mu \mathrm{m}$, and the slices were stained with hematoxylin and eosin (H\&E) for light microscopy.

\section{Statistical analysis}

All data are presented as mean \pm standard deviation (SD) and were analyzed by one-way ANOVA using SPSS 19.0 (IBM SPSS Statistics, USA). Differences were considered to be statistically significant at $p<0.05$.

\section{RESULTS}

\section{KSOL decreased RI and UP level in rats with GS-induced AKI}

As shown in Table 1, the RI and UP level in the GS group were increased significantly, compared with the NS group $(p<0.01)$, indicating that the AKI model was successfully established with GS treatment. Furthermore, these increases were reversed by treatment with furosemide or UCG, suggesting that the injuries could be reversed successfully with known treatments. After treatment with $\mathrm{KSOL}(20,10$, or $5 \mathrm{~mL} / \mathrm{kg})$, the RI and UP level were decreased significantly relative to the GS group $(p<0.01)$, suggesting that the KSOL offered an improvement to AKI injury in this model.

\section{KSOL down-regulated $\mathrm{SCr}$ and BUN levels in rats with GS-induced AKI}

As shown in Table 2, the SCr and BUN levels in the GS group were increased significantly, compared with the NS group $(p<0.01)$, indicating that the AKI model had been successfully established. After treatment with UCG or KSOL $(20,10$, or $5 \mathrm{~mL} / \mathrm{kg})$, the SCr level was decreased significantly, compared with the GS group $(p<0.01)$. After treatment with UCG or $\mathrm{KSOL}(10$ or $5 \mathrm{~mL} / \mathrm{kg}$ ), the BUN level was decreased significantly relative to the GS group $(p<0.05$ or 0.01$)$. 
Table 1: KSOL decreased RI and UP level in rats with GS-induced AKI

\begin{tabular}{lccc}
\hline Group & Dose/kg & $\mathbf{R I}$ & UP $(\mathbf{m g} / \mathbf{L})$ \\
\hline NS & - & $0.799 \pm 0.040^{* *}$ & $616.300 \pm 144.000^{* *}$ \\
GS & - & $1.200 \pm 0.030$ & $1702.800 \pm 140.300$ \\
Furosemide & $6.6 \mathrm{mg}$ & $1.100 \pm 0.098^{* *}$ & $1373.600 \pm 241.100^{* *}$ \\
UCG & $4.2 \mathrm{~g}$ & $1.030 \pm 0.081^{* *}$ & $1373.000 \pm 188.900^{* *}$ \\
High KSOL & $20 \mathrm{~mL}$ & $1.079 \pm 0.089^{* *}$ & $1533.000 \pm 175.800^{* *}$ \\
Middle KSOL & $10 \mathrm{~mL}$ & $1.028 \pm 0.085^{* *}$ & $1589.400 \pm 129.600^{* *}$ \\
Low KSOL & $5 \mathrm{~mL}$ & $1.029 \pm 0.068^{* *}$ & $1513.600 \pm 175.100^{* *}$ \\
\hline
\end{tabular}

All data stood for mean \pm standard deviation; ${ }^{* *} p<0.01$, compared with the GS group

Table 2: KSOL down-regulated SCr and BUN levels in rats with GS-induced AKI

\begin{tabular}{lccc}
\hline Group & Dose $/ \mathbf{k g}$ & $\mathbf{S C r}(\boldsymbol{\mu m o l} / \mathbf{L})$ & BUN $(\mathbf{m m o l} / \mathbf{L})$ \\
\hline NS & - & $24.411 \pm 5.678^{\star *}$ & $9.463 \pm 2.324^{\star *}$ \\
GS & - & $79.383 \pm 16.854$ & $32.200 \pm 6.822$ \\
Furosemide & $6.6 \mathrm{mg}$ & $68.238 \pm 18.134$ & $28.515 \pm 7.953$ \\
UCG & $4.2 \mathrm{~g}$ & $57.533 \pm 11.994^{* *}$ & $24.235 \pm 4.208^{* *}$ \\
High KSOL & $20 \mathrm{~mL}$ & $59.684 \pm 13.816^{\star *}$ & $24.663 \pm 10.488$ \\
Middle KSOL & $10 \mathrm{~mL}$ & $53.016 \pm 9.612^{* *}$ & $24.417 \pm 6.951^{*}$ \\
Low KSOL & $5 \mathrm{~mL}$ & $55.382 \pm 13.140^{\star *}$ & $21.339 \pm 4.092^{\star *}$ \\
\hline
\end{tabular}

All data stood for mean \pm standard deviation; ${ }^{*} p<0.05,{ }^{* *} p<0.01$, compared with the GS group

Effect of KSOL on MDA level, NAG activity and SOD activity in rats with GS-induced AKI

As depicted in Table 3, MDA level and NAG activity in GS group were increased significantly, and the SOD activity was reduced significantly, compared with the NS group $(p<0.01)$. These data indicated that the AKI model was successfully established. After treatment with furosemide, UCG, or KSOL $(20,10$, or $5 \mathrm{~mL} / \mathrm{kg}$ ), the MDA level and NAG activity were reduced significantly, compared with the GS group $(p<$ 0.05 or 0.01 ). After treatment with UCG or KSOL $(20,10$, or $5 \mathrm{~mL} / \mathrm{kg})$, the SOD activity was increased significantly, compared with the GS group $(p<0.05$ or 0.01$)$.

\section{Effect of KSOL on histopathological characteristics of kidney tissue of rats with GS-induced AKI}

In the NS group, glomeruli, renal tubules, and collecting tubules were normal (Figure $1 \mathrm{~A}$ and
B). In the GS group, the glomeruli were atrophied, and the epithelial cells of the tubules had extreme edema, which resulted in stenosis of the lumens. There was severe lymphocytic infiltration in the interstitium of the cortex. Significant protein deposits were observed in the collecting tubules, and the renal interstitium was hyperemic (Figure $1 \mathrm{C}$ and $\mathrm{D}$ ).

In the furosemide groups, the glomeruli were normal, but the epithelial cells of tubules exhibited edema. Protein deposits were observed in the renal tubules and severe lymphocytic infiltration was observed in the interstitium of the cortex. Protein deposits had accumulated in the collecting tubules, and the renal interstitium was hyperemic (Figure $1 \mathrm{E}$ and F). In the UCG group, the histological sections were similar to those in the furosemide group, except that the renal interstitium exhibited extreme hyperemia (Figure $1 \mathrm{G}$ and $\mathrm{H}$ ).

Table 3: Effect of KSOL on NAG, MDA and SOD in rats with GS-induced AKI

\begin{tabular}{lcccc}
\hline Group & Dosage/ kg & NAG (U/L) & $\begin{array}{c}\text { MDA } \\
\text { (nmol/mg prot) }\end{array}$ & $\begin{array}{c}\text { SOD } \\
\text { (U/mg prot) }\end{array}$ \\
\hline NS & - & $3.626 \pm 0.720^{\star *}$ & $0.785 \pm 0.116^{\star *}$ & $239.000 \pm 63.1^{\star \star}$ \\
GS & - & $7.257 \pm 0.886$ & $1.384 \pm 0.392$ & $162.800 \pm 19.000$ \\
Furosemide & $6.6 \mathrm{mg}$ & $6.134 \pm 1.452^{\star}$ & $0.963 \pm 0.356^{\star}$ & $186.900 \pm 38.900$ \\
UCG & $4.2 \mathrm{~g}$ & $4.167 \pm 0.846^{\star \star}$ & $0.814 \pm 0.199^{\star \star}$ & $209.100 \pm 43.300^{\star \star}$ \\
High KSOL & $20 \mathrm{~mL}$ & $5.881 \pm 0.698^{\star *}$ & $0.972 \pm 0.387^{\star}$ & $195.800 \pm 25.900^{\star \star}$ \\
Middle KSOL & $10 \mathrm{~mL}$ & $5.811 \pm 1.084^{\star \star}$ & $0.810 \pm 0.178^{\star \star}$ & $191.000 \pm 32.900^{\star}$ \\
Low KSOL & $5 \mathrm{~mL}$ & $6.015 \pm 1.374^{\star}$ & $0.901 \pm 0.298^{\star \star}$ & $188.600 \pm 34.600^{\star}$ \\
\hline
\end{tabular}

All data stood for mean \pm standard deviation; ${ }^{*} p<0.05,{ }^{* *} p<0.01$, compared with the GS group 


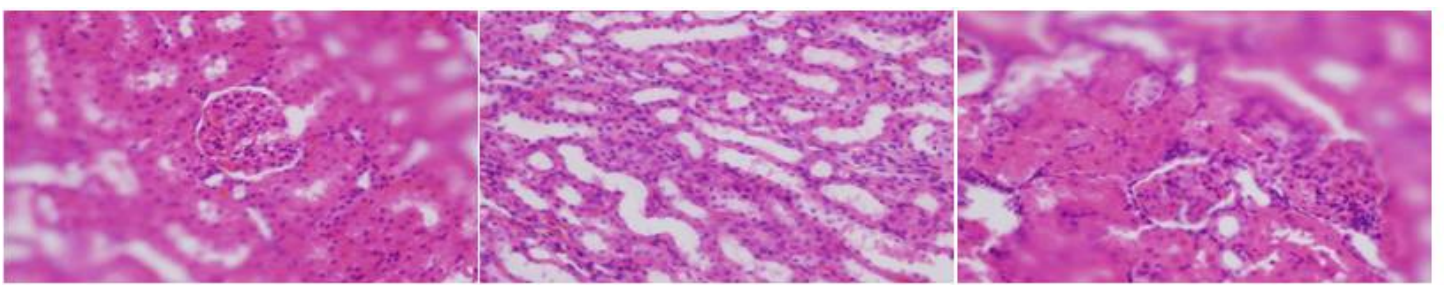

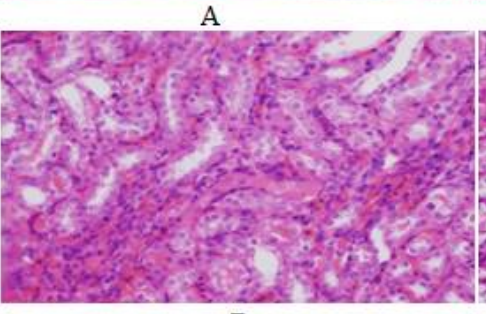

$\mathrm{D}$

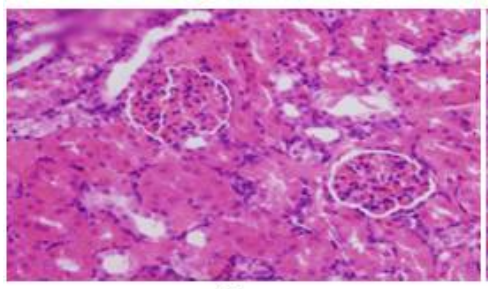

G

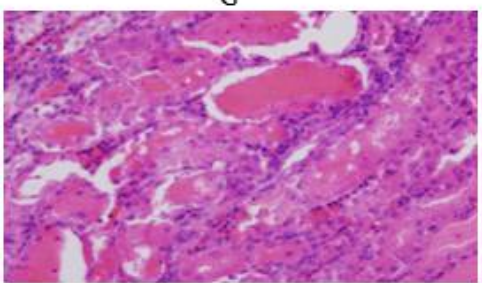

$\mathrm{J}$

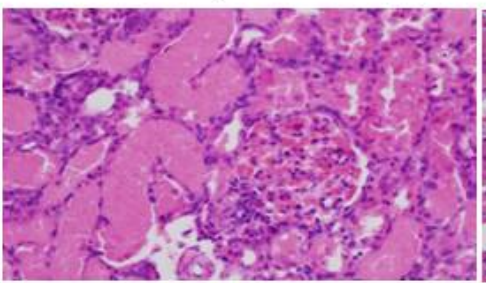

M
B

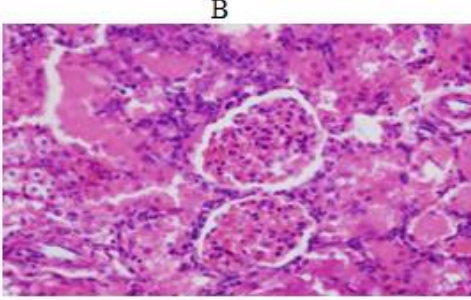

E

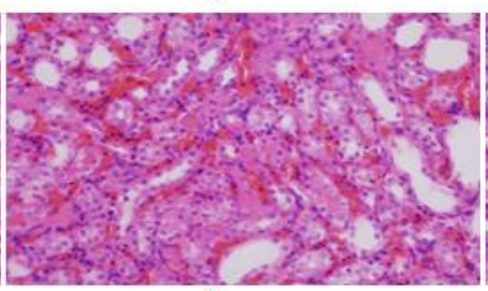

$\mathrm{H}$

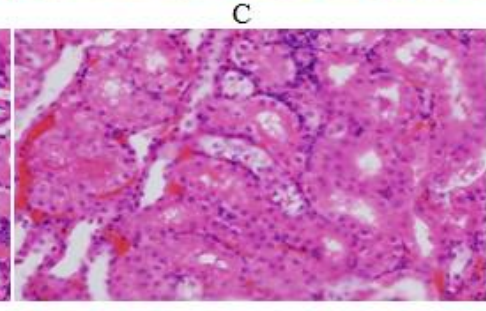

F

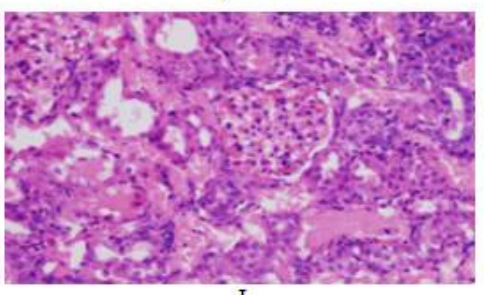

I

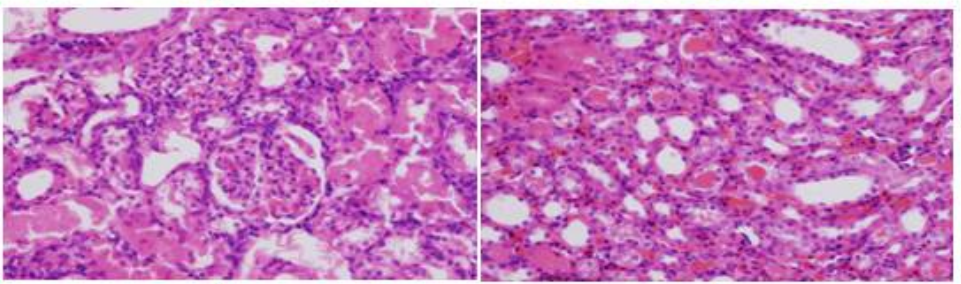

$\mathrm{K}$

L

Figure 1: Effects of KSOL on histopathological appearance in the kidney tissues of rats with GS-induced AKI; A and B: Kidney tissues from the NS group; C and D: Kidney tissues from the GS group; E and F: Kidney tissues from the furosemide group; $\mathrm{G}$ and $\mathrm{H}$ : Kidney tissues from the UCG group; I and J: Kidney tissues from the high dose of KSOL group; $\mathrm{K}$ and L: Kidney tissues from the middle dose of $\mathrm{KSOL}$ group, $\mathrm{M}$ and $\mathrm{N}$ : Kidney tissues from the low dose of KSOL group; (haematoxylin \& eosin staining, magnification $\times 400$ )

In the high-dose KSOL group, the histological sections were similar to those from the furosemide group (Figure $1 \mathrm{I}$ and $\mathrm{J}$ ). In the middle dose of KSOL group, the histological sections were similar to those from the furosemide group, except that there were some protein deposits in the renal tubules and slight lymphocytic infiltration in the interstitium of the cortex (Figure $1 \mathrm{~K}$ and $\mathrm{L}$ ). In the low dose of KSOL group, the histopathological characteristics were similar to those observed in the UCG group (Figure 1M and $\mathrm{N}$ ).

\section{DISCUSSION}

Administration of GS is considered an acceptable model to induce AKI in rats. Upon GS administration, several physiological markers are used to measure the severity of AKI, including RI and UP levels, NAG activity in urine, serum $\mathrm{SCr}$ BUN levels, and renal MDA level; all of these increased under conditions of AKI. Additionally, SOD activity is decreased in rat kidney with AKI [17-19]. We observed all of these physiological changes in our AKI model. Moreover, many histopathological characteristics, including atrophy of the glomeruli, lymphocytic infiltration in 
the interstitium of the cortex, protein deposits in the collecting tubules, and hyperemia of the renal interstitium, can be observed in rat kidney with GS-induced AKI [17-19].

In the present study, we investigated the effect of $\mathrm{KSOL}$ on GS-induced $\mathrm{AKI}$ in rats by measuring these various AKI-related indices and histopathological changes. We confirmed that GS can induce all of these changes in this rat model of AKI. Furosemide and UCG each offer a protective effect on the kidney, and thus served as positive control treatments [20,21].

Several physiological indices can be used to evaluate the overall health of the kidney. $\mathrm{Rl}$ is a simple index to reflect edema of the kidney, and an increase in $\mathrm{Rl}$ indicates that edema of the kidney has occurred [22]. UP can reflect filtration function of the kidney, where kidney damage is reflected by an increase in UP level [19]. Although glomerular filtration rate (GFR) is the best overall index of renal function in health and disease [23], it is difficult to measure in clinical practice. Therefore, most clinicians estimate the GFR based on the SCr level [24]. Renal parenchymal injury can lead to an increase in BUN level [19].

NAG, a lysosomal enzyme whose molecular weight does not permit filtration through glomeruli, exists in the proximal tubular cells. An increase in NAG activity in the urine indicates that kidney dysfunction has occurred [25]. MDA level can assess lipid peroxidation, which can induce tissue damage through oxygen-free radicals [26]. An increase in MDA level in kidney tissue indicates that lipid peroxidation has occurred. SOD is an essential defense against the damage of superoxide [27].

The mechanism of GS-induced nephrotoxicity seems to be a result of destructive reactive oxygen species, which can participate in kidney injury [19]. Thus, increase in SOD activity can accelerate recovery from AKI. In this study, KSOL exhibited a protective effect on GSinduced $\mathrm{AKI}$ in rats at the biochemical level by increasing the activity of SOD and reducing the $\mathrm{RI}$, activity of NAG, and levels of UP, SCr, BUN and MDA.

Moreover, our results show that KSOL improved GS-induced histopathological characteristics in the kidney tissue of rats with $\mathrm{AKI}$, including atrophy of the glomeruli, lymphocytic infiltration in the interstitium of the cortex, protein deposits in the collecting tubules, and hyperemia of the renal interstitium.

\section{CONCLUSION}

KSOL has a protective effect on GS-induced AKI in rats by increasing the activity of SOD and decreasing RI, NAG activity, as well as levels of UP, SCr, BUN and MDA. KSOL also improves the histopathological profile of kidneys. Thus, $\mathrm{KSOL}$ is capable of preventing the toxic effects of GS on kidneys at biochemical and histopathological levels, and thus has a potential to be developed into a therapeutic treatment for AKI.

\section{CONFLICT OF INTEREST}

The authors declare that there is no conflict of interest associated with this work.

\section{CONTRIBUTION OF AUTHORS}

We declare that this work was done by the authors named in this article and all liabilities pertaining to claims relating to the content of this article will be borne by the authors. Jing-Sheng Zhao, Wei-Guo Yi and Xiang-Nan Li conceived and designed the study. Jing-Sheng Zhao, YuanLiang Wei, Xin-Wei Lin, Hong Liu, Xue-Qing Xiao, Jun Yang and Dan Hao performed the experiments, collected and analyzed the data.

\section{REFERENCES}

1. Kellum JA, Unruh ML, Murugan R. Acute kidney injury. BMJ Clin Evid 2011; 2011: 2001.

2. Bellomo R, Kellum JA, Ronco C. Acute kidney injury. Lancet 2012; 380(9843): 756-766.

3. Ralib A, Mat-Nor MB. Acute kidney injury in a Malaysian intensive care unit: Assessment of incidence, risk factors, and outcome. J Crit Care 2015; 30(3): 636-642.

4. Cartin-Ceba R, Kashiouris M, Plataki M, Kor DJ, Gajic O, Casey ET. Risk factors for development of acute kidney injury in critically ill patients: a systematic review and meta-analysis of observational studies. Crit Care Res Pract 2012; 2012: 691013.

5. Finlay $S$, Bray $B$, Lewington AJ, Hunter-Rowe $C T$, Banerjee A, Atkinson JM, Jones MC. Identification of risk factors associated with acute kidney injury in patients admitted to acute medical units. Clin Med 2013; 13(3): 233-238.

6. Medve L, Antek C, Paloczi B, Kocsi S, Gartner B, Marjanek Z, Bencsik G, Kanizsai $P$, Gondos $T$. Epidemilogy of acute kidney injury in hungarian intensive care units: a multicenter, prospective, observational study. BMC Nephrol 2011; 12(1): 43.

7. Uchino S, Kellum MD, Bellomo R, Doig GS, Morimatsu H, Morgera S, Scheta M, Tan I, Bouman C, Macedo E, Gibney N, Tolwani A, Ronco C. Acute renal failure in 
critically III patients: a multinational, multicenter study. JAMA 2005; 294(4): 1203-1210.

8. Liaño $F$, Junco E, Pascual J, Madero R, Verde E. The spectrum of acute renal failure in the intensive care unit compared with that seen in other settings. The Madrid Acute Renal Failure Study Group. Kidney Int Suppl 1998; 66: S16-24.

9. Hou SH, Bushinsky DA, Wish JB, Cohen JJ, Harrington JT. Hospital-acquired renal insufficiency: a prospective study. Am J Med 1983; 74(2): 243-248.

10. Nash K, Hafeez A, Hou S. Hospital-acquired renal insufficiency. Am J Kidney Dis 2002; 39(5): 930-936.

11. Mei CL, Liu SY. Advances in diagnosis and treatment of acute kidney injury. Med J Chin PLA 2013; 38(5): 342346.

12. Coca SG, Yusuf B, Shlipak MG, Garg AX, Parikh CR. Long-term risk of mortality and other adverse outcomes after acute kidney injury: a systematic review and metaanalysis. Am J Kidney Dis 2009; 53(6): 961-973.

13. The National Research Council of The National Academy of Sciences. Guide for the Care and Use of Laboratory Animals: Eight Edition. Washington, D.C.: The National Academies Press; 2010.

14. Sedmak JJ, Grossberg SE. A rapid, sensitive, and versatile assay for protein using coomassie brilliant blue G250. Anal Biochem 1977; 79(1-2): 544-552.

15. Walker $P D$, Shah SV. Evidence suggesting a role for hydroxyl radical in gentamicin-induced acute renal failure in rats. J Clin Invest 1988; 81(2): 334-341.

16. Polat A, Parlakpinar H, Tasdemir S, Colak C, Vardi N, Ucar M, Emre MF. Protective role of amino guanidine on gentamicin-induced acute renal failure. Acta histochem 2006; 108(5): 365-371.

17. An YX, Tang H. Experimental study on antagonism of tetrandrine in acute renal injury induced by gentamicin in guinea pig. Clin J Appl Physiol 2003; 19(3): 278-281.
18. Fan WM, Wang XQ, Liu CF. Effect of Zuoguiwan on the expression of MKK4, MKK 7 and JNK in rats with gentamicin-induced renal injury. Chin $J$ Integr Tradit West Nephrol 2013; 14(2): 100-103.

19. Qiu YL, Hong M, Fu X, Huang HX, Ma J. Urine biomarkers after acute kidney injury in rats induced by gentamycin. Chin J Pharmacol Toxicol 2014; 28(2): 248254.

20. Ho KM, Power BM. Benefits and risks of furosemide in acute kidney injury. Anaesthesia 2010; 65(3): 283-293.

21. Wu HL, Lin HC, Ruan XL, Deng CH, She YP, Fang JA. Observation of the curative efficiency of uremic clearance granules in 118 patients with chronic renal failure. Chin J Integr Tradit West Nephrol 2004; 5(1): 21 24.

22. Ji H, Li Z, Po ZM, Chen F, Chen PS. Effects of cultural mycelium of Cordyceps sinensis (CMCs) on gentamycin-induced acute kidney injury in rats. J Chin Pharmaceut Univ 1996; 27(4): 245-249.

23. Levey AS. Measurement of renal function in chronic renal disease. Kidney Int 1990; 38(1): 167-184.

24. Levey AS, Bosch JP, Lewis JB, Greene T, Rogers N, Roth $D$. A more accurate method to estimate glomerular filtration rate from serum creatinine: $A$ new prediction equation. Ann Intern Med 1999; 130(6): 461-470.

25. Erdem A, Gündoğan A, Usubütün, $K$ Kilinc, Erdem $S R$, Kara A, Bozkurt $A$. The protective effect of taurine against gentamicin-induced acute tubular necrosis in rats. Nephrol Dial Transp 2000; 15(8): 1175-1182.

26. Wong STY, Knight JA, S M Hopfer, O Zaharia, Leach CN $J r$, Sunderman FW Jr. Lipoperoxides in plasma as measured by lipid-chromatographic separation of malondialdehyde-thiobarbituric acid adduct. Clin Chem 1987; 33(2): 214-220.

27. Beauchamp C, Fridovich I. Superoxide dismutase: Improved assays and an assay applicable to acrylamide gels. Anal Biochem 1971; 44(1): 276-287. 